\title{
The effects of movement stimulation on activities of daily living performance and quality of life in nursing home residents with dementia: a randomized controlled trial
}

This article was published in the following Dove Press journal:

Clinical Interventions in Aging

\author{
Marinda Henskens' \\ Ilse M Nauta ${ }^{2}$ \\ Katja T Drost ${ }^{3}$ \\ Erik JA Scherder' \\ 'Department of Clinical \\ Neuropsychology, Vrije Universiteit \\ Amsterdam, Amsterdam, the \\ Netherlands; ${ }^{2}$ Department of \\ Neurology, MS Center Amsterdam, \\ Vrije Universiteit Medical Center, \\ Amsterdam, the Netherlands; \\ ${ }^{3}$ tanteLouise, Bergen op Zoom, \\ the Netherlands
}

\begin{abstract}
Background: Nursing home $(\mathrm{NH})$ residents with dementia experience a reduced quality of life (QoL), in part, due to a dependence in performing activities of daily living (ADL). Stimulating movement is associated with improvements in ADL performance. Therefore, movement stimulating interventions, such as ADL training and exercise, focus on optimizing ADL performance to improve QoL. This study aimed to evaluate the effect of three movement stimulating interventions on QoL and ADL performance in NH residents with dementia.
\end{abstract}

Methods: In this 6-month double parallel randomized controlled trial, the effects of ADL training, a multicomponent aerobic and strength exercise training, and a combined ADL and exercise training were analyzed in $87 \mathrm{NH}$ residents with dementia. The Global Deterioration Scale was used to classify the severity of dementia. Participants were screened at baseline using the 6 minute walk test and Mini-Mental State Examination. The Qualidem, and the Care Dependency Scale and Erlangen ADL test were evaluated at baseline, and after 3 and 6 months to measure QoL and ADL, respectively. Multilevel analyses were adjusted for baseline performance, age, and gender.

Results: A 6-month ADL training positively affected overall QoL $(p=0.004)$ and multiple aspects of QoL, including care relationship ( $p=0.004)$, positive self-image $(p=0.002)$, and feeling at home $(p=0.001)$, compared to care-as-usual. No benefits were observed of exercise on QoL. No benefits were observed of a combined ADL and exercise intervention on QoL. No effects were found of the three movement interventions on ADL performance.

Conclusion: The results indicate that ADL training can improve QoL. The results contribute to the limited knowledge regarding the effect of movement stimulation on resident outcomes. Further large-scale studies are recommended.

Keywords: dementia, exercise, institutionalization, activities of daily living, quality of life

\section{Introduction}

One-third to two-thirds of elderly with dementia live in nursing homes (NHs). ${ }^{1-3}$ Currently, there is no cure for dementia, and non-pharmacological interventions are aimed at improving a residents' quality of life (QoL). ${ }^{4}$ Dichter et $\mathrm{al}^{5}$ define dementia-specific QoL as "the multidimensional evaluation of the person-environment system of the individual, in terms of adaptation to the perceived consequences of the dementia".

NH residents with dementia show a significantly lower QoL compared to homedwelling elderly with dementia. ${ }^{6}$ This may be the result of poorer physical function and dependence in activities of daily living (ADL) often seen in NH residents with
Correspondence: Marinda Henskens Department of Clinical Neuropsychology, Vrije Universiteit Amsterdam, Van der Boechorststraat I, I08I BT Amsterdam, the Netherlands

Tel +3I 205988769

Email m.henskens@vu.nl 
dementia. ${ }^{7-9}$ Independence in ADL is a key determinant of QoL, ${ }^{10}$ and therefore, non-pharmacological interventions, such as ADL training and exercise, focus on stimulating movement to optimize ADL performance and improve QoL. ${ }^{4}$

Previous studies have shown that movement stimulation is associated with improvements in physical performance (eg, muscle strength and endurance) and ability to perform ADLs (eg, bathing and dressing) among elderly with dementia. ${ }^{1-15}$ Despite these benefits, the majority of $\mathrm{NH}$ residents with dementia are inactive ${ }^{16}$ and limited in the opportunities to participate in physical activity in the NH.

Two methods are commonly described in the literature to stimulate movement in the NH: ADL training and exercise training. ADL training focuses on stimulating movement during daily care tasks, by encouraging the resident to perform as much of their self-care as independently as possible. ${ }^{17}$ However, evidence of the benefits of ADL training on ADL performance is inconsistent. While some studies show positive effects, ${ }^{17-21}$ others find no benefits. ${ }^{22-26}$ This inconsistency may be justified by a smaller sample size and higher levels of cognitive impairment observed in non-effective studies. ${ }^{23,25}$ With regard to QoL, one study found a higher positive self-image after a 12-month ADL training compared to care-as-usual. ${ }^{27}$ To our knowledge, no other studies found improvements in QoL following ADL training. ${ }^{19}$

The other method to stimulate movement in the $\mathrm{NH}$, exercise training, often consists of aerobic exercises, strength training, or a combination of both. While recent studies provide some support for the benefits of exercise on ADL performance, ${ }^{11,14,28}$ the studies varied greatly in duration ( 2 weeks to 12 months), type (aerobic, strength, and balance), and frequency (weekly to daily). ${ }^{11}$ While the largest improvements were reported after 6-month multicomponent interventions ${ }^{14}$ with a frequency of 3 times per week, ${ }^{29,30}$ definite conclusions cannot be made due to the limited number of high-quality studies and heterogeneity of interventions. ${ }^{29}$ A multicomponent intervention is thought to yield the greatest benefits, as aerobic and strength training can each benefit cognition by enhancing cerebral blood flow. ${ }^{31,32}$ Furthermore, strength training can improve skills necessary for ADLs (eg, mobility and balance). ${ }^{33}$ Evidence for the effectiveness of exercise programs on QoL is limited for $\mathrm{NH}$ residents with dementia, presumably due to the methodological limitations of subjective and proxy ratings. ${ }^{34}$

A previous study compared the effect of ADL training to exercise training. They found a slower decline in ADL performance following both interventions compared to the control group. ${ }^{21}$ It would be interesting to investigate the effect of a combined ADL and exercise intervention, since a combination may yield more benefits than ADL or exercise alone. Even though there are multiple valuable studies that investigate movement interventions in $\mathrm{NH}$ residents with dementia, it remains unclear which type of movement stimulation is best for improving QoL and ADL performance. ${ }^{14}$

The purpose of this study was, therefore, to compare the effects of three movement stimulation interventions on QoL and ADL performance among NH residents with dementia. Specifically, we investigated the effect of 1) ADL training, 2) a multicomponent exercise training (aerobic and strength), and 3) a combined ADL and exercise training. To our knowledge, this is the first study to combine an ADL and exercisebased intervention in $\mathrm{NH}$ residents with dementia. It was hypothesized that a 6-month ADL or exercise intervention results in a maintenance, or a slower decline of QoL and ADL performance compared to residents receiving care-as-usual. A combined ADL and exercise training was expected to result in the greatest benefits on QoL and ADL performance.

\section{Methods Design}

A 6-month double parallel randomized controlled trial with three movement stimulation interventions was conducted among NH residents with dementia. This study contains aspects of a pragmatic trial, as interventions are carried out in a real-world clinical setting, with typical patients and qualified clinicians. ${ }^{35}$ Assessors of the Erlangen ADL (E-ADL) test were blinded for the group allocations. Due to the nature of our study, it was not possible to blind the participants and their first responsible caregivers. This trial was prospectively registered at the Dutch Trial registration (NTR5641).

\section{Randomization}

Eleven locations of a long-term care organization ("tanteLouise") in the Netherlands were randomized to an ADL training or care-as-usual (control) intervention. For each location, participants were individually randomized to an exercise or social activity (control) intervention, resulting in four groups: 1) PADL: physical activity and ADL training, 2) SADL: social activity and ADL training, 3) PCO: physical activity and care-as-usual, and 4) SCO: social activity and care-as-usual (Figure 1). An independent researcher randomized the locations and the participants. For the latter, a random number generator was used with random block sizes and random number of blocks. 


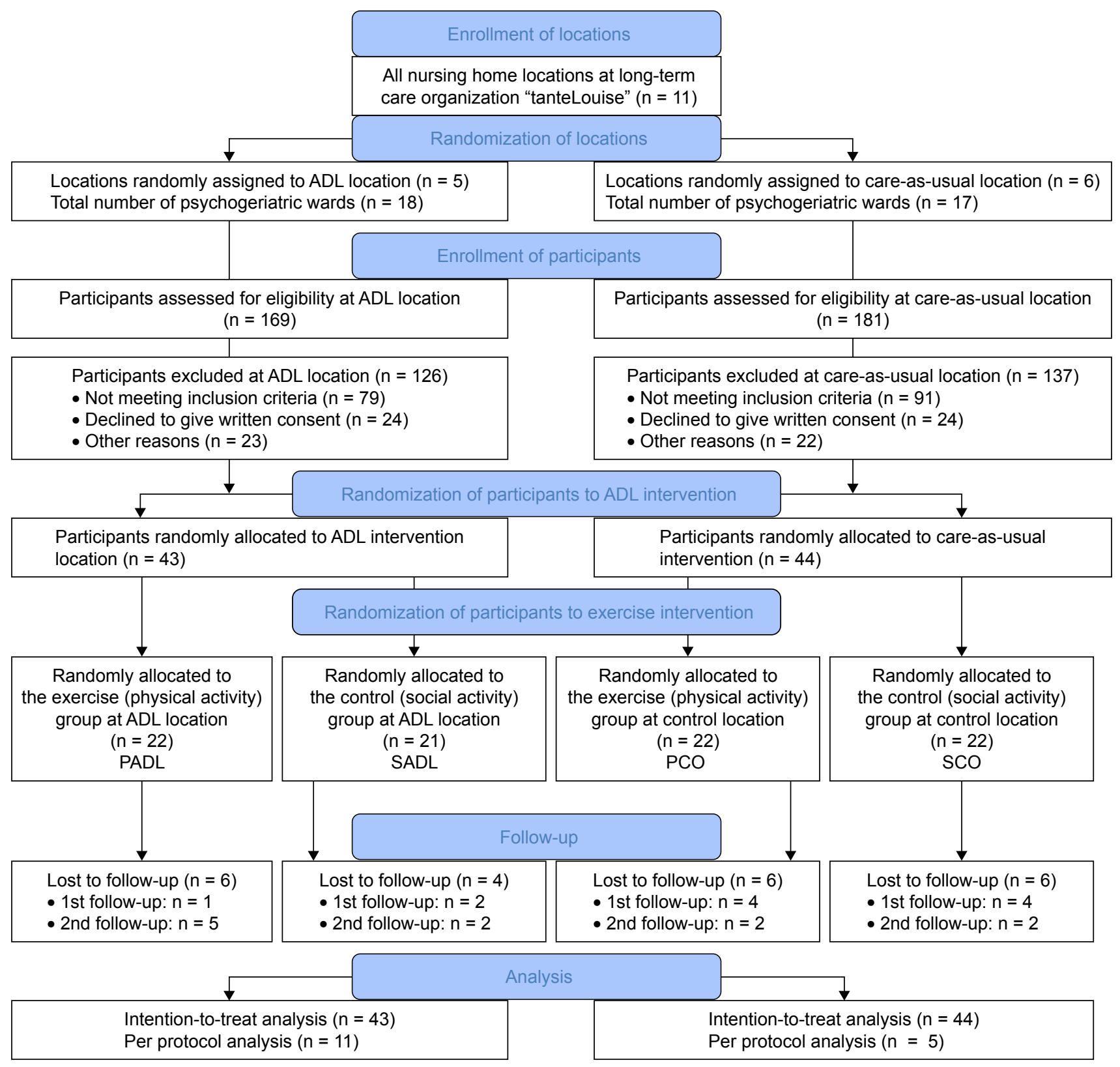

Figure I Flowchart of the sampling procedure.

Abbreviations: ADL, activities of daily living; PADL, physical activity and ADL; SADL, social activity and ADL; PCO, physical activity and control; SCO, social activity and control.

\section{Participants}

A flow diagram of the sampling procedure is represented in Figure 1. Eighty-seven NH residents with dementia were included in the study. Inclusion criteria were: 1) living in a psychogeriatric ward of NH "tanteLouise", 2) a diagnosis of dementia according to the Diagnostic and Statistical Manual of Mental Disorders, 4th ed, ${ }^{36} 3$ ) age $\geq 65$ years, 4) a score on the Mini-Mental State Examination (MMSE) $)^{37}$ between 1 and 24, and 5) able to complete the 6-minute walking test (6MWT) (with or without walking aid). ${ }^{38}$ Exclusion criteria were: 1) wheelchair bound, 2) very poor vision, 3) severe cardiovascular problems, 4) instable diabetes mellitus, or 5 ) aggressive or runaway behavior. The dropout rate was not significantly different among the four groups, $\chi^{2}=0.57$, $p=0.89$.

\section{Ethics approval and informed consent}

The Medical Ethics Review Committee of VU University Medical Center Amsterdam (2015.446) concluded that this study was not subject to the Medical Research Involving Human Subjects Act (WMO). Approval was granted by the Scientific and Ethical Review Board (VCWE) of the 
Faculty of Behavior \& Movement Sciences of Vrije Universiteit Amsterdam (VCWE-2015-183R1). Written informed consent was obtained from the legal representatives of the participants.

\section{Demographics}

Information regarding the type of dementia was derived from the patients' medical charts. Education level was classified according to the system of Verhage, ${ }^{39}$ with scores ranging from 1 ( $<6$ years of primary education) to 7 (academic degree). The Global Deterioration Scale (GDS) ${ }^{40}$ was used to classify the severity of dementia, with scores ranging from 1 (no cognitive decline) to 7 (very severe cognitive decline). GDS was determined by the participants' medical doctors. A short version of the Severe Impairment Battery (SIB-S) was used as a performance-based assessment of low-level cognitive skills, ${ }^{41}$ with scores ranging from 0 to 50 . The SIB-S is reliable for elderly with MMSE scores below $5 .^{41}$

\section{Materials and procedure \\ Materials}

\section{Screening instruments}

The 6-minute walk test $(6 \mathrm{MWT})^{38}$ was used to measure aerobic fitness, determined by the distance walked in meters. The $\mathrm{MMSE}^{37}$ was used to determine global cognition. Scores range from 0 to 30, with a score of 23 or lower indicating dementia.

ADL

ADL was assessed with the Care Dependency Scale (CDS $)^{42}$ and the E-ADL test. ${ }^{43}$ The CDS consists of 15 items concerning the level of independence. Scores ranged from 15 to 75 , with higher scores representing a higher level of independence. The CDS is completed by the participants' first responsible caregiver in $<5$ minutes. The CDS is reliable (Cronbach's alpha 0.97; inter-rater reliability 0.51-0.71) (22,44 $^{4}$ and has a strong hierarchical scale ( $H$-coefficient 0.75$).{ }^{42}$ The E-ADL is a performance test in which residents are asked to perform five basic ADL tasks. Scores range from 0 to 30, with higher scores representing better ADL performance. The E-ADL is administered by trained neuropsychology students who were blind to the intervention, and takes less than 10 minutes to complete. The E-ADL has a high validity and a good reliability (Cronbach's alpha 0.77; test-retest reliability 0.73). ${ }^{43} \mathrm{An}$ "ADL" domain was created, which is the sum of the $z$-scores of the CDS and E-ADL.

\section{QoL}

QoL was assessed with the Qualidem, ${ }^{45,46}$ consisting of 37 items rated on a four-point rating scale, ranging from 0 (never) to 3 (often). The questionnaire consists of nine subscales: 1) care relationship (range 0-21), 2) positive affect (range 0-18), 3) negative affect (range 0-9), 4) restless tense behavior (range 0-9), 5) positive self-image (range 0-9), 6) social relations (range 0-18), 7) social isolation (range 0-9), 8) feeling at home (range 0-12), and 9) having something to do (range 0-6). For each subscale, a higher score represents a better QoL. The overall QoL score is the sum of the $z$-scores of the nine subscales. The Qualidem is completed by the participants' first responsible caregiver in $\sim 10$ minutes. The Qualidem is sufficiently reliable (Cronbach's alpha 0.59-0.89; inter-rater reliability $0.47-0.79),{ }^{45,47}$ and most subscales show moderate to strong scalability, with the exception of social isolation which shows weak scalability (Loevinger's coefficient $H<0.4$ ). ${ }^{47}$

\section{Procedure}

Physiotherapists provided functional mobility assessments of all the residents using the Arjo Mobility Gallery. ${ }^{48}$ Only residents with mobility levels A or B (able to walk and perform ADLs independently, with or without some assistance) were included (see inclusion and exclusion criteria). Accordingly, geriatricians gave advice about the medical and physical capabilities of the residents. After written informed consent was granted, participants were screened for inclusion and randomized to the exercise or control group. Outcome variables were measured at baseline, and after 3 and 6 months.

\section{Interventions}

\section{ADL training}

In this 6-month individually based intervention, nursing staff were asked to stimulate movement during daily care tasks by encouraging residents to perform as much of their self-care as independently as possible throughout the day. Examples are encouraging a resident to participate in bathing or dressing, or having a resident set the table (see Table 1 for an example of an individually based health care plan). Nursing staff could provide verbal cues to assist the resident. By encouraging independence during daily (care) tasks, it was possible to focus on improving specific ADL functions, and to stimulate movement throughout the whole day. For each ward per ADL location, ambassadors (consisting of one physio- or occupational therapist and two nursing staff) were appointed to receive three 3 -hour educational sessions by qualified physio- and occupational therapists. Consequently, ambassadors were responsible for sharing their knowledge about ADL stimulation with the other nursing staff. The individual nature of the intervention allowed for variance in training between participants based on their capabilities and limitations. Individually based goals, plans, capabilities, and 
Table I Example of an individualized health care plan

\begin{tabular}{ll}
\hline Domain & Example from a participant who could walk independently \\
\hline Living conditions & Client likes to help with household tasks and is stimulated to help as much as possible. Client tidies up her apartment herself, \\
but her family does the laundry. Sometimes client wants to wash her own clothes; if this is the case, stimulate her to do so. \\
She has laundry detergent in her room. Her bedsheets are washed weekly by the service agency, but client is encouraged to \\
change her bed herself. Client can independently prepare breakfast and is stimulated to get the newspaper in the morning. \\
Client likes to go outside to walk. Stimulate client to participate in day activities. Involve client in household tasks such as \\
preparing meals, setting and clearing the table, and folding napkins. Client takes care of groceries independently. Client likes \\
to be social, but needs to be stimulated to do so. \\
Client has some problems with getting out of bed at night, sometimes resulting in bedwetting. This makes client anxious and \\
restless. Assist client at night with going to the toilet between 0I:00 am and 02:00 am and between 05:00 am and 06:00 am \\
in the morning. \\
Client is able to do a lot independently, sometimes with help of verbal cues. Remind client to brush her teeth, including \\
the denture. Client can wash herself independently, but remind her to wash her face and arms. Assist in choosing clothes, \\
Physical well- \\
being and health
\end{tabular}

activities were reported in four domains of the health care plan: 1) living conditions, 2) participation, 3) mental wellbeing and autonomy, and 4) physical well-being and health (Table 1). Participants in the control locations received careas-usual and completed all outcome measures at the same time as the intervention group.

\section{Exercise training}

The 6-month multicomponent exercise intervention consisted of strength and aerobic exercises, with a frequency of three times per week, for 30-45 minutes per session. Sessions were alternated weekly, in which one week consisted of two strength sessions and one aerobic session, followed by a week of two aerobic sessions and one strength session. Strength exercises focused on lower extremities (eg, squats, calf raises), upper extremities (eg, chest press, biceps/triceps curl), and the torso (eg, seated back extension, chair crunch). Exercises were performed in three sets of eight repetitions, which could be increased to $10-12$ or 15 repetitions only after correct and pain-free execution. Additional increase in intensity could be achieved by adding weights. The aerobic training consisted of outdoor walking sessions. Residents were assigned to either a short walking route $(500 \mathrm{~m})$ or a long route $(1 \mathrm{~km})$, based on their performance on the 6MWT. The aerobic sessions had a moderate to intensive intensity. The NH provided qualified movement teachers who guided the small-scale strength and aerobic exercise groups (4-6 residents). Nursing staff were asked to drink tea with the control group three times per week to control for the social aspect of the exercise intervention.

\section{Compliance to the intervention}

Twenty-three ambassadors from five ADL locations were asked to fill out a monthly online questionnaire to determine compliance to the ADL intervention. Attendance to the exercise intervention, and reasons for absence, were reported weekly for each participant.

\section{Statistical analysis}

Statistical analyses were performed using IBM SPSS 21.0. Differences between groups in demographic characteristics and outcome variables at baseline were analyzed using one way independent ANOVA and Pearson's chi-square tests. Analyses were performed using a modified intention-to-treat approach including all participants with at least one postbaseline assessment. Multilevel analyses were performed using a three-level model (observations [level 1] nested within participants [level 2] nested within NHs [level 3]). An advantage of multilevel analysis is that it is flexible in handling missing data. ${ }^{49}$ Data were analyzed using two models: the first model examined the overall intervention effects, independent of time, while the second model examined the difference in intervention effects over time. The ADL group (PADL and SADL) was compared to the care-as-usual control group (PCO and SCO) to examine the effect of ADL training. The exercise group (PADL and PCO) was compared to the social activity control group (SADL and SCO) to examine the effect of exercise. The combined ADL and exercise group (PADL) was compared to the group receiving no movement stimulation (SCO) to examine the effect of a combined ADL and exercise intervention. Data were analyzed using a crude model (adjusted for baseline performance) and an adjusted model (adjusted for baseline performance, age, and gender). Statistical significance was determined as $p<0.05$. For the Qualidem scales, a Bonferroni correction was used to correct for alpha inflation $(p<0.005[0.05 / 10])$. Regression coefficients were reported, representing an overall intervention 
effect for model 1 (group estimate), and the intervention effect at different time points for model 2 (group-by-time estimate). Per protocol analyses were performed including only participants who attended at least $75 \%$ of the exercise sessions.

\section{Power analysis}

With a power of $80 \%$ and an alpha of 0.05 , it is possible to detect a change of 0.48 SDs in the outcome variable with a comparison between two groups with 120 participants.

\section{Results}

\section{Comparisons at baseline}

We intended to include 120 participants from the 350 residents living at NH "tanteLouise". We were able to include 87 participants due to inclusion and exclusion criteria, and because some participants expressed unwillingness to participate. The demographics of the participants are listed in Table 2. At baseline, the PCO group walked longer distances than the SCO group $(p=0.019)$ and the PADL group ( $p=0.002)$. No significant differences were found between groups on baseline ADL and QoL (all $p$ 's $>$ 0.05 , Table 3$)$. The 22 participants who dropped out were significantly older ( 88 vs $85 ; p=0.05$ ) and had a higher GDS (5.63 vs 5.19; $p<0.05)$ than the participants who completed the study. Although participants with a MMSE between 2 and 5 had a lower average SIB score than participants with a MMSE between 6 and 23 (24.6 vs 42.0), the mean SIB scores indicated that all participants were capable of following instructions.

\section{Compliance to the intervention Exercise}

Of the 36 strength and 36 aerobic training sessions, participants attended an average of 20 strength (56\%) and 19 aerobic (54\%) sessions. The 16 participants who attended more than $75 \%$ of the intervention, attended an average of 30 strength (83\%) and 29 aerobic sessions (81\%). Main reasons for noncompliance were influenza, norovirus, lack of motivation, overlapping activities, and bank holidays.

ADL

Table 4 presents a summary of the questionnaires. The total response rate was $76 \%$. Sixty-nine percent of the time, nursing staff succeeded in not taking over care tasks which residents could perform independently. The extent to which staff succeeded in stimulating residents to perform more ADLs independently was $66 \%$. The extent to which ambassadors motivated other staff to stimulate residents was $70 \%$. Reasons for non-compliance were that nursing staff forgot to stimulate ADL (6\%), had a high work pressure $(14 \%)$, or thought it was faster to do it themselves (9\%). Other reasons were fatigue (54\%), unwillingness $(45 \%)$, or lack of understanding (14\%) of the resident. To a lesser extent, ambassadors indicated that ADL was not stimulated at all times because staff had too little knowledge regarding the

Table 2 Mean and SD of demographic characteristics of participants at baseline $(N=87)$

\begin{tabular}{|c|c|c|c|c|}
\hline Characteristics & PADL $(n=22)$ & SADL $(n=2 I)$ & $\operatorname{PCO}(n=22)$ & $\operatorname{SCO}(n=22)$ \\
\hline Age (years), mean (SD) & $86.95(7.21)$ & $86.05(5.86)$ & $85.14(4.64)$ & $84.73(4.55)$ \\
\hline Age, range & $75-100$ & $7 \mid-95$ & $75-93$ & $75-94$ \\
\hline Gender (female), n (\%) & 14 (63.4\%) & $19(90.5 \%)$ & 17 (77.3\%) & 17 (77.3\%) \\
\hline \multicolumn{5}{|l|}{ Education } \\
\hline Low (I-3) & $8(36.4 \%)$ & $9(42.9 \%)$ & 8 (36.4\%) & $3(13.6 \%)$ \\
\hline Medium (4-5) & $12(54.5 \%)$ & $9(42.9 \%)$ & $10(45.5 \%)$ & II (50\%) \\
\hline High (6-7) & $2(9.1 \%)$ & $2(9.5 \%)$ & $\mathrm{I}(4.5 \%)$ & $3(13.6 \%)$ \\
\hline GDS, mean (SD) & $5.05(0.80)$ & $5.19(0.84)$ & $5.40(0.82)$ & $5.55(0.83)$ \\
\hline GDS, range & $3-6$ & $4-6$ & $3-6$ & $3-6$ \\
\hline SIB-S, mean (SD) & $42.55(6.63)$ & $40.90(5.97)$ & $37.82(9.96)$ & $38.55(9.27)$ \\
\hline MMSE, mean (SD) & I3.55 (5.6I) & $13.19(3.67)$ & $12.14(6.43)$ & $10.23(5.67)$ \\
\hline 6MWT, mean $(\mathrm{SD})^{*}$ & |76.7| (72.24) & 207.34 (7I.72) & $253.12(65.67)$ & $191.55(58.36)$ \\
\hline \multicolumn{5}{|l|}{ Diagnosis, n (\%) } \\
\hline Alzheimer's disease (AD) & $6(27.3 \%)$ & $6(28.6 \%)$ & $14(63.6 \%)$ & $8(36.4 \%)$ \\
\hline Vascular dementia (VD) & $3(13.6 \%)$ & $5(23.8 \%)$ & I (4.5\%) & $3(13.6 \%)$ \\
\hline Mixed, VD and AD & $3(13.6 \%)$ & I (4.8\%) & I (4.5\%) & I (4.5\%) \\
\hline Other/unknown & $10(45.5 \%)$ & $9(42.9 \%)$ & $6(27.3 \%)$ & $9(40.9 \%)$ \\
\hline
\end{tabular}

Note: ${ }^{*} p<0.05$.

Abbreviations: MMSE, Mini-Mental State Examination; 6MWT, 6-minute walk test; GDS, Global Deterioration Scale; ADL, activities of daily living; PADL, physical activity and ADL; SADL, social activity and ADL; PCO, physical activity and control; SCO, social activity and control; SIB-S, Severe Impairment Battery short version. 


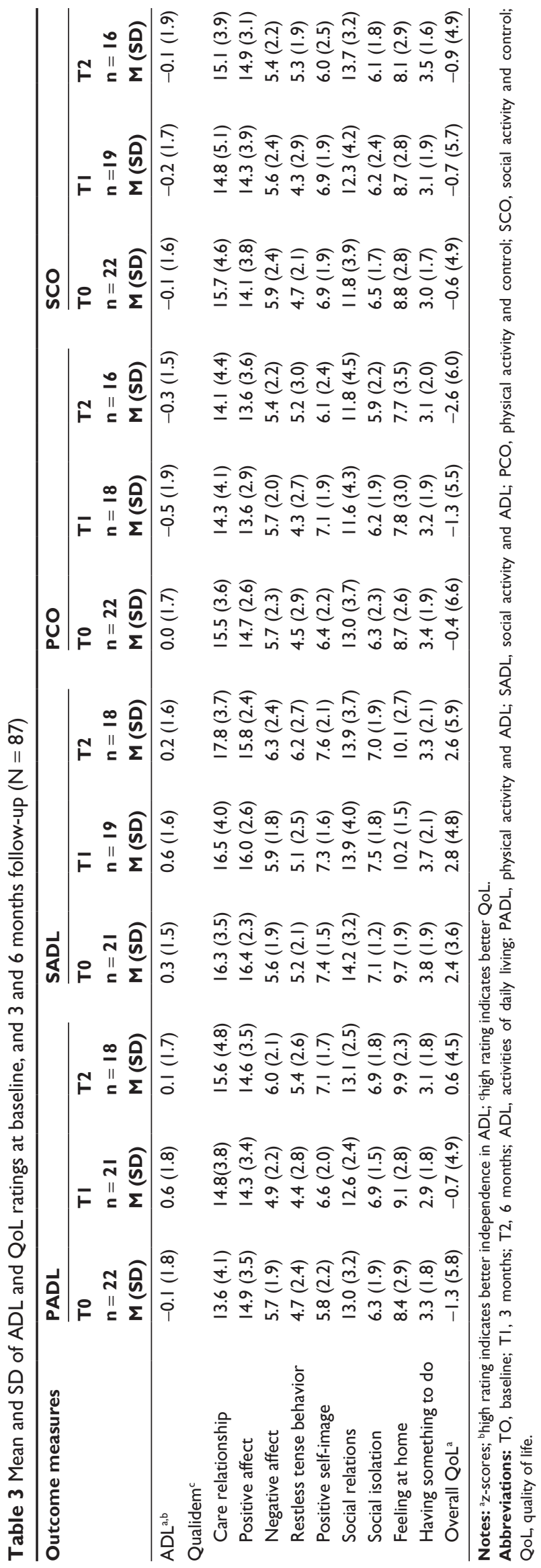

importance of stimulation (5.5\%) or had too little guidance on how to stimulate ADL (5.5\%).

\section{Intention-to-treat analysis}

\section{Effect of ADL training}

Crude and adjusted multilevel models providing overall intervention effects are presented in Table 5. There was a significant main effect of ADL training on the QoL domain feeling at home ( $p=0.002$ ), indicating that on average over time, the ADL group (PADL and SADL) scored significantly higher than the care-as-usual group (PCO and SCO). In addition, there was a significant main effect on overall QoL $(p=0.02)$ and care relationship $(p=0.009)$ which disappeared after adjusting for alpha inflation. Table 6 shows the effect of the intervention after 3 and 6 months. Significant group-by-time interactions were found for QoL following the ADL training. Specifically, after 6 months, the ADL group scored significantly higher than the care-as-usual group on care relationship ( $p=0.004)$ adjusted for baseline scores, and overall QoL ( $p=0.004)$, positive self-image $(p=0.002)$, and feeling at home $(p=0.001)$ adjusted for baseline scores, age, and gender. There was no significant main effect or group-by-time effect of ADL training on ADL performance. However, we did observe a maintenance in ADL performance in the ADL group, compared to a decline in the care-as-usual group. This difference was not significant.

\section{Effect of exercise training}

No significant main effects were found on QoL and ADL performance following the exercise training compared to the control group (PCO and PADL vs SADL and SCO; Table 5). No significant group-by-time interactions were found in QoL or ADL performance (Table 6).

\section{Effect of a combined ADL and exercise training}

No significant main effects were found on QoL and ADL performance following the combined ADL and exercise intervention (PADL vs SCO; Table 5). After 6 months, the PADL group scored significantly higher than $\mathrm{SCO}$ on positive self-image $(p=0.007)$ and feeling at home $(p=0.02)$, adjusted for baseline performance. However, these group-bytime interactions were no longer significant after adjusting for alpha inflation (Table 6).

\section{Per protocol analysis}

Subgroup analyses were performed including only participants who attended at least $75 \%$ of the exercise sessions $(n=16[36 \%])$ to examine the effect of the exercise 
Table 4 Monthly results of compliance to the ADL intervention

\begin{tabular}{|c|c|c|c|c|c|c|}
\hline Research question & $\begin{array}{l}\text { TI } \\
\text { M (\%) }\end{array}$ & $\begin{array}{l}\text { T2 } \\
\text { M (\%) }\end{array}$ & $\begin{array}{l}\text { T3 } \\
\text { M (\%) }\end{array}$ & $\begin{array}{l}\text { T4 } \\
\text { M (\%) }\end{array}$ & $\begin{array}{l}\text { T5 } \\
\text { M (\%) }\end{array}$ & $\begin{array}{l}\text { T6 } \\
\text { M (\%) }\end{array}$ \\
\hline $\begin{array}{l}\text { To what extent did you manage to stimulate the participants to perform } \\
\text { more ADL activities independently? (scale } 0-100 \text { ) }\end{array}$ & 58.3 & 60 & 68 & 67 & 81 & 68 \\
\hline $\begin{array}{l}\text { To what extent did you succeed in motivating other staff members to } \\
\text { stimulate residents to perform ADL activities independently? (scale } 0-100 \text { ) }\end{array}$ & 52 & 66 & 74 & 72 & 87 & 74 \\
\hline $\begin{array}{l}\text { To what extent did you take over care tasks that residents were able to } \\
\text { perform independently? (scale } 0-100 \text { ) }\end{array}$ & 29 & 37 & 26 & 29 & 37 & 28 \\
\hline
\end{tabular}

Abbreviations: $T$, time in months; $A D L$, activities of daily living; $M$, mean.

intervention. No significant differences were observed between the exercise group (PADL and PCO) compared to the control group (SADL and SCO) regarding QoL and ADL performance (all $p$ 's $>0.05$ ).

\section{Discussion}

The present study examined the effect of ADL training, exercise, and a combined ADL and exercise training on QoL and ADL performance in $\mathrm{NH}$ residents with dementia. We found clinically relevant results of the ADL intervention, as we demonstrate that a 6-month ADL training positively affects overall QoL and multiple aspects of QoL, including positive self-image, care relationship, and feeling at home, when compared with care-as-usual. The improvements in selfimage suggest that residents who received ADL training show less signs of worthlessness, ${ }^{5}$ a feeling frequently experienced in elderly with dementia. ${ }^{50}$ The daily limitations which $\mathrm{NH}$ residents with dementia face can negatively impact their selfimage and QoL, highlighting the relevance of maintaining a positive self-image.$^{51}$ Improvements in care relationship

Table 5 Results of multilevel analyses regarding the effect of ADL training, exercise training, and the combined training (PADL) on QoL and ADL performance

\begin{tabular}{|c|c|c|c|c|c|c|c|}
\hline \multirow[t]{2}{*}{ Outcome measures } & \multirow[t]{2}{*}{ Model } & \multicolumn{2}{|l|}{$\begin{array}{l}\text { ADL vs control }{ }^{a} \\
n=44 / 43\end{array}$} & \multicolumn{2}{|c|}{$\begin{array}{l}\text { Exercise vs control } \\
n=43 / 44\end{array}$} & \multicolumn{2}{|l|}{$\begin{array}{l}\text { PADL vs SCO } \\
n=22 / 22\end{array}$} \\
\hline & & Beta $(95 \% \mathrm{Cl})$ & $p$-value & Beta $(95 \% \mathrm{Cl})$ & $p$-value & Beta $(95 \% \mathrm{Cl})$ & $p$-value \\
\hline \multirow[t]{2}{*}{ ADL } & Cru & $0.4(-0.2,0.9)$ & 0.16 & $-0.3(-0.8,0.2)$ & 0.24 & $0.1(-0.7,0.8)$ & 0.90 \\
\hline & Adj & $0.4(-0.1,0.9)$ & 0.13 & $-0.5(-0.9,0.1)$ & 0.09 & $-0.0(-0.8,0.7)$ & 0.90 \\
\hline \multicolumn{8}{|l|}{ Qualidem } \\
\hline \multirow[t]{2}{*}{ Care relationship } & Cru & $2.0(0.5,3.5)$ & $0.009 *$ & $-0.2(-1.8,1.3)$ & 0.76 & I.8 $(-0.4,3.9)$ & 0.11 \\
\hline & Adj & $2.0(0.5,3.6)$ & $0.01 *$ & $-0.2(-1.8,1.5)$ & 0.86 & $1.8(-0.4,4.1)$ & 0.11 \\
\hline \multirow[t]{2}{*}{ Positive affect } & Cru & $0.5(-0.6,1.7)$ & 0.34 & $-0.6(-1.7,0.5)$ & 0.26 & $-0.1(-1.6,1.4)$ & 0.92 \\
\hline & Adj & $0.7(-0.5,1.8)$ & 0.24 & $-0.4(-1.5,0.8)$ & 0.51 & $0.3(-1.3,1.8)$ & 0.73 \\
\hline \multirow[t]{2}{*}{ Negative affect } & Cru & $0.3(-0.4$, I.I $)$ & 0.39 & $-0.3(-I . I, 0.5)$ & 0.47 & $0.1(-0.9,1.2)$ & 0.88 \\
\hline & Adj & $0.4(-0.4, I . I)$ & 0.34 & $-0.5(-1.2,0.3)$ & 0.21 & $-0.1(-1.2,0.9)$ & 0.83 \\
\hline \multirow[t]{2}{*}{ Restless tense behavior } & Cru & $0.2(-1.2,1.6)$ & 0.81 & $-0.2(-I . I, 0.7)$ & 0.65 & $0.2(-1.1,1.4)$ & 0.81 \\
\hline & Adj & $0.2(-1.2,1.6)$ & 0.73 & $-0.2(-1.1,0.7)$ & 0.64 & $0.2(-1.1,1.5)$ & 0.76 \\
\hline \multirow[t]{2}{*}{ Positive self-image } & Cru & $0.6(-0.1,1.2)$ & 0.09 & $0.3(-0.4,0.9)$ & 0.39 & $0.9(-0.1,1.8)$ & 0.08 \\
\hline & Adj & $0.6(-0.1,1.3)$ & 0.08 & $0.2(-0.5,0.9)$ & 0.61 & $0.8(-0.2,1.7)$ & 0.12 \\
\hline \multirow[t]{2}{*}{ Social relations } & Cru & $0.3(-0.9,1.5)$ & 0.63 & $-0.6(-1.8,0.6)$ & 0.30 & $-0.4(-2.0,1.3)$ & 0.68 \\
\hline & Adj & $0.4(-0.8,1.7)$ & 0.51 & $-0.5(-1.7,0.8)$ & 0.44 & $-0.1(-1.8,1.6)$ & 0.93 \\
\hline \multirow[t]{2}{*}{ Social isolation } & Cru & $0.7(-0.6,2.0)$ & 0.25 & $0.0(-0.7,0.7)$ & 0.97 & $0.9(-0.2,2.0)$ & 0.10 \\
\hline & Adj & $0.8(-0.6,2.1)$ & 0.23 & $0.1(-0.6,0.8)$ & 0.79 & I.I $(-0.1,2.2)$ & 0.07 \\
\hline \multirow[t]{2}{*}{ Feeling at home } & Cru & I.7 $(0.7,2.7)$ & $0.002^{* *}$ & $-0.5(-1.4,0.5)$ & 0.33 & I.3 $(-0.2,2.7)$ & 0.08 \\
\hline & Adj & $1.7(0.7,2.7)$ & $0.002 * *$ & $-0.5(-1.5,0.5)$ & 0.31 & $1.2(-0.3,2.7)$ & 0.11 \\
\hline \multirow[t]{2}{*}{ Having something to do } & Cru & $-0.3(-0.9,0.3)$ & 0.26 & $-0.1(-0.7,0.5)$ & 0.82 & $-0.4(-1.2,0.5)$ & 0.36 \\
\hline & Adj & $-0.3(-0.9,0.3)$ & 0.33 & $-0.0(-0.6,0.6)$ & 0.99 & $-0.2(-1.2,0.6)$ & 0.51 \\
\hline \multirow[t]{2}{*}{ Overall QoL } & Cru & $2.2(0.3,4.1)$ & $0.02 *$ & $-0.7(-2.6,1.2)$ & 0.45 & I.6 (-I.I, 4.3) & 0.23 \\
\hline & Adj & $2.4(0.5,4.4)$ & $0.02 *$ & $-0.5(-2.5,1.4)$ & 0.59 & $1.9(-0.9,4.7)$ & 0.17 \\
\hline
\end{tabular}

Notes: ${ }^{a}$ Control group, care-as-usual; ${ }^{b}$ control group, social activity group; ${ }^{*} p<0.05,{ }^{* *} p<0.005$. Cru: adjusted for baseline scores; Adj: adjusted for baseline scores, gender, and age.

Abbreviations: ADL, activities of daily living; QoL, quality of life; PADL, physical activity and ADL; SCO, social activity and control. 


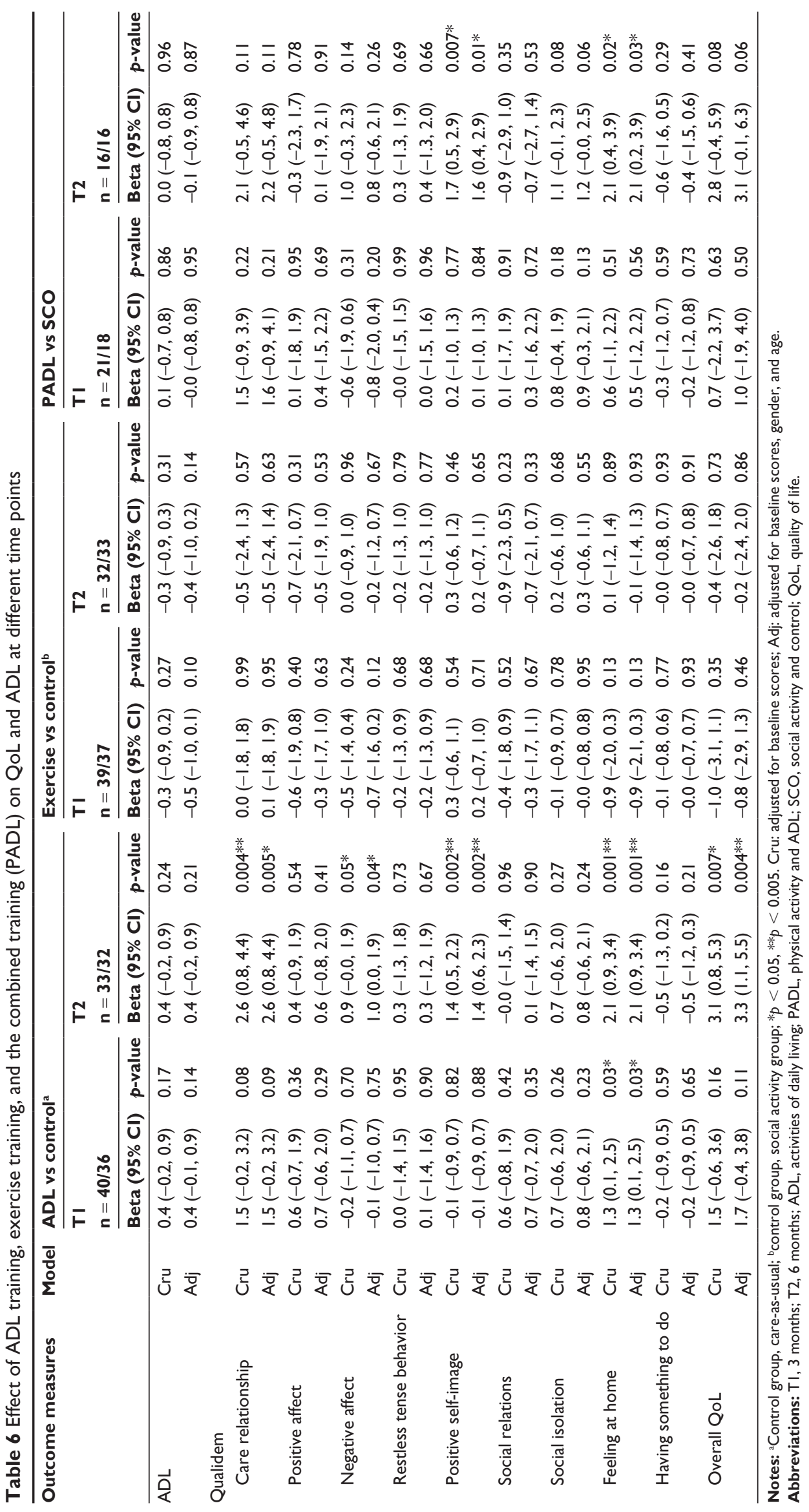


(eg, manifested by residents being more prone to accept help) and feeling at home are crucial for a successful adaptation to the $\mathrm{NH}$ and the well-being of a resident. ${ }^{52}$ In order to improve care relationship, staff must provide residents with more control, autonomy, and trust. ${ }^{53,54}$ Having an adequate care relationship is an important factor that influences residents' sense of feeling at home, and an inadequate care relationship may result in aggressive or uncooperative behavior. ${ }^{53}$ Moreover, performing familiar activities from one's previous home-life (eg, domestic chores) is found to positively influence feeling at home. ${ }^{53}$ It is possible that the ADL training improved care relationship by providing the resident with control, autonomy, and extra attention throughout the day. Consequently, improvements in care relationship, in combination with the focus of the ADL training on stimulating participation in domestic chores and self-care, may have played a role in the improved sense of feeling at home.

Improvements in QoL may also be related to ADL performance, which is a key determinant of QoL. ${ }^{10}$ Although no effects were found of ADL training on ADL performance, we did observe a trend that showed a maintenance in ADL performance in the ADL group, and a decline in the care-asusual group. Although these differences were not significant, maintenance in ADL is considered a positive finding, as $\mathrm{NH}$ residents with dementia typically experience a decline in ADL performance over a 6-month period. ${ }^{55}$

The second movement intervention, multicomponent exercise, did not benefit QoL or ADL performance, contrary to our expectations. Although exercise shows promising effects on QoL in healthy elderly, ${ }^{56}$ lack of benefits regarding QoL in our study is in line with previous studies conducted in the NH population. ${ }^{34,57}$ We particularly expected multicomponent exercise training to benefit QoL by improving or maintaining independent functioning. ${ }^{11,14,15,28,30,58}$ Previous studies frequently report improvements in lower extremity skills (eg, transfers and walking stairs) following multicomponent exercise interventions. ${ }^{15,30}$ However, we did not find benefits of a multicomponent exercise intervention on ADL performance. Perhaps no improvements were found in ADL because the exercise training focused more on lower extremity skills, while the ADL instruments focused more on upper extremity skills (eg, cutting bread or washing hands). Only two items of the ADL instruments in the present study measured lower extremity skills (eg, mobility and posture). Another explanation may be the lower adherence to the intervention (55\%) compared to similar studies (>75\%), ${ }^{11,14,15,59}$ as adherence to the intervention is found to be an important predictor of change in ADL. ${ }^{30}$ Reasons for low adherence were infections, overlapping activities (eg, music programs), and bank holidays, which may be more common in longer interventions. ${ }^{30}$ Nonetheless, per protocol analyses of participants who attended at least $75 \%$ of the sessions also did not show benefits of exercise. Perhaps a higher intervention frequency is required to achieve improvements in ADL, as previous studies found improvements after 120 minutes of aerobic and strength exercises per week, independent of the duration of the intervention (12 weeks to 12 months). ${ }^{15,30,59}$

The third movement intervention, combined ADL and exercise training, did not benefit QoL or ADL performance any more than ADL or exercise training alone. This could indicate that only ADL training is beneficial for $\mathrm{NH}$ residents with dementia. However, the study may also be underpowered to detect differences between these smaller subgroups.

\section{Strengths and limitations}

The strengths of this study include the use of outcome measures specifically designed for elderly with dementia. In addition, the Qualidem is the only dementia-specific QoL instrument that considers care relationship and feeling at home in the $\mathrm{NH}$, which are important indicators for a successful adaptation to the NH. ${ }^{60}$ Furthermore, this study has a randomized design, which is a frequently reported limitation in previous studies that include NH residents with dementia. ${ }^{34}$

Some limitations should be considered. First, it was not possible to blind the nursing staff who completed the CDS and Qualidem, potentially leading to observer bias. Second, assessment of QoL should consider the multidimensionality of QoL. The subjective (emotional well-being), objective (social, economic, and environmental), and functional (goalrelated values) components of QoL make it a difficult concept to measure. ${ }^{34,61}$ The Qualidem considers this multidimensionality as it includes aspects of psychological well-being (eg, positive and negative affect) as well as the objective environment (eg, living environment and contact with caregivers). Despite the multidimensionality of the Qualidem, an instrument that combines subjective, objective, and functional components of QoL may provide the most complete indication of dementia-specific QoL. ${ }^{61}$

A third limitation is that calculating an overall QoL score might lead to loss of information due to the varying content of the subscales. ${ }^{45}$ Although we applied a Bonferroni correction to correct for alpha inflation, interpreting an overall QoL score should be done with caution.

Fourth, we did not register the amount of physical activity outside the sessions. Differences in activity levels between the groups outside of the sessions may have influenced QoL 
or ADL. Last, the overall sample size may have been too small to provide sufficient statistical power to show significant differences. Nevertheless, these findings are of value to the limited number of studies investigating the effect of ADL and exercise interventions in NH residents with dementia.

\section{Conclusion}

To our knowledge, this is the first study to combine an ADL and exercise training in NH residents with dementia. We conclude that ADL training is beneficial for overall QoL and multiple aspects of QoL. The exercise training was not effective in improving QoL or ADL performance. There were no additional benefits of a combined ADL and exercise intervention, suggesting that ADL training alone is enough to improve QoL. These findings are clinically relevant, since this trial indicates that QoL of $\mathrm{NH}$ residents with dementia can be improved in daily clinical practice. Ensuring QoL is a major goal of dementia care, ${ }^{4}$ however, the amount of research in this area is limited due to the methodological challenges of measuring QoL, such as heterogeneity in outcome measures (proxy vs self-report). ${ }^{34}$ Therefore, our study adds to the limited knowledge regarding the effect of ADL and exercise training on QoL and ADL performance. The effectiveness of an ADL intervention in the NH could lead to improved care for $\mathrm{NH}$ residents with dementia. Further largescale studies are required to draw more firm conclusions.

\section{Data sharing statement}

The data that support the findings of this study are available from Stichting tanteLouise, but restrictions apply to the availability of these data, which were used under license for the current study, and so are not publicly available. Data are available from the authors upon reasonable request and with permission of Stichting tanteLouise.

\section{Acknowledgments}

This study is funded by Stichting tanteLouise, who contributed to the conception and design of the study, helped with data acquisition, and critically revised the manuscript. The study funders had no role in the analysis and interpretation of the data.

\section{Author contributions}

$\mathrm{MH}$ conducted the study, supervised data collection, performed the data analysis and interpretation, and writing of the manuscript. IMN designed and conducted the study, supervised data collection, and contributed to the data analysis and writing of the manuscript. KTD advised in designing and conducting the study, contributed to data collection, and critically revised the manuscript. EJAS advised in designing the study, contributed to interpretation of data, and critically revised the manuscript. All authors read and approved the final manuscript.

\section{Disclosure}

The authors report no conflicts of interest in this work.

\section{References}

1. Macdonald A, Cooper B. Long-term care and dementia services: an impending crisis. Age Ageing. 2007;36(1):16-22.

2. Alzheimer's Association. 2013 Alzheimer's disease facts and figures. Alzheimer's Dement. 2013;9(2):208-245.

3. Alzheimer Europe. Appendix 1: The prevalence of dementia in Europe. In: Dementia in Europe Yearbook 2013. Luxembourg: Alzheimer Europe; 2013:978-972.

4. Yasuda M, Sakakibara H. Care staff training based on person-centered care and dementia care mapping, and its effects on the quality of life of nursing home residents with dementia. Aging Ment Health. 2017; 21(9):991-996.

5. Dichter MN, Ettema TP, Schwab CG, et al. QUALIDEM User Guide. Witten, Amsterdam: DZNE/VUmc; 2016.

6. Olsen C, Pedersen I, Bergland A, et al. Differences in quality of life in home-dwelling persons and nursing home residents with dementia-a cross-sectional study. BMC Geriatr. 2016;16(1):137.

7. Schüssler S, Dassen T, Lohrmann C. Comparison of care dependency and related nursing care problems between Austrian nursing home residents with and without dementia. Eur Geriatr Med. 2015;6(1):46-52.

8. Wetzels R, Zuidema SU, de Jonghe JF, Verhey F, Koopmans R. Determinants of quality of life in nursing home residents with dementia. Dement Geriatr Cogn Dis. 2010;29(3):189-197.

9. Zuidema SU, Derksen E, Verhey FR, Koopmans RT. Prevalence of neuropsychiatric symptoms in a large sample of Dutch nursing home patients with dementia. Int J Geriatr Psychiatry. 2007;22(7):632-638.

10. Ballard C, O'Brien J, James I, et al. Quality of life for people with dementia living in residential and nursing home care: the impact of performance on activities of daily living, behavioral and psychological symptoms, language skills, and psychotropic drugs. Int Psychogeriatr. 2001;13(1):93-106.

11. Forbes D, Thiessen EJ, Blake CM, Forbes SC, Forbes S. Exercise programs for people with dementia. Cochrane Database Syst Rev. 2013; 12:CD006489.

12. Brett L, Traynor V, Stapley P. Effects of physical exercise on health and well-being of individuals living with a dementia in nursing homes: a systematic review. JAMDA. 2016;17(2):104-116.

13. Lee HS, Park SW, Park YJ. Effects of physical activity programs on the improvement of dementia symptom: a meta-analysis. BioMed Res Int. 2016;2016.

14. Rao AK, Chou A, Bursley B, Smulofsky J, Jezequel J. Systematic review of the effects of exercise on activities of daily living in people with Alzheimer's disease. Am J Occup Ther. 2014;68(1):50-56.

15. Santana-Sosa E, Barriopedro MI, Lopez-Mojares LM, Perez M, Lucia A. Exercise training is beneficial for Alzheimer's patients. Int J Sports Med. 2008;29(10):845-850.

16. van Alphen HJM, Volkers KM, Blankevoort CG, Scherder EJA, Hortobágyi T, van Heuvelen MJG. Older adults with dementia are sedentary for most of the day. PLoS ONE. 2016;11(3):e0152457.

17. Resnick B, Gruber-Baldini AL, Zimmerman S, et al. Nursing home resident outcomes from the res-care intervention. J Am Geriatr Soc. 2009;57(7):1156-1165.

18. Galik E, Resnick B, Hammersla M, Brightwater J. Optimizing function and physical activity among nursing home residents with dementia: testing the impact of function-focused care. Gerontologist. 2013; 54(6):930-943. 
19. Resnick B, Galik E, Boltz M. Function focused care approaches: literature review of progress and future possibilities. J Am Med Dir Assoc. 2013;14(5):313-318.

20. Chang S-H, Wung S-F, Crogan NL. Improving activities of daily living for nursing home elder persons in Taiwan. JNR. 2008;57(3): 191-198.

21. Morris JN, Fiatarone M, Kiely DK, et al. Nursing rehabilitation and exercise strategies in the nursing home. J Gerontol A Biol Sci Med Sci. 1999;54(10):M494-M500.

22. Talley KM, Wyman JF, Savik K, Kane RL, Mueller C, Zhao H. Restorative care's effect on activities of daily living dependency in long-stay nursing home residents. Gerontologist. 2015;55(Suppl 1):S88-S98.

23. Galik E, Resnick B, Lerner N, Hammersla M, Gruber-Baldini AL. Function focused care for assisted living residents with dementia. Gerontologist. 2015;55(Suppl 1):S13-S26.

24. Resnick B, Galik E, Gruber-Baldini A, Zimmerman S. Testing the effect of function-focused care in assisted living. J Am Geriatr Soc. 2011; 59(12):2233-2240.

25. Galik EM, Resnick B, Gruber-Baldini A, Nahm E-S, Pearson K, Pretzer-Aboff I. Pilot testing of the restorative care intervention for the cognitively impaired. J Am Med Dir Assoc. 2008;9(7):516-522.

26. Kerse N, Peri K, Robinson E, et al. Does a functional activity programme improve function, quality of life, and falls for residents in long term care? Cluster randomised controlled trial. BMJ. 2008;337:a1445.

27. Henskens M, Nauta IM, Scherder EJA, Oosterveld FGJ, Vrijkotte S. Implementation and effects of movement-oriented restorative care in a nursing home - a quasi-experimental study. BMC Geriatr. 2017;17(1):243.

28. Littbrand H, Stenvall M, Rosendahl E. Applicability and effects of physical exercise on physical and cognitive functions and activities of daily living among people with dementia: a systematic review. Am J Phys Med Rehabil. 2011;90(6):495-518.

29. Blankevoort CG, Van Heuvelen MJ, Boersma F, Luning H, De Jong J, Scherder EJ. Review of effects of physical activity on strength, balance, mobility and ADL performance in elderly subjects with dementia. Dement Geriatr Cogn Disord. 2010;30(5):392-402.

30. Rolland Y, Pillard F, Klapouszczak A, et al. Exercise program for nursing home residents with Alzheimer's disease: a 1-year randomized, controlled trial. J Am Geriatr Soc. 2007;55(2):158-165.

31. Nation DA, Hong S, Jak AJ, et al. Stress, exercise, and Alzheimer's disease: a neurovascular pathway. Med Hypotheses. 2011;76(6):847-854.

32. Özkaya GY, Aydin H, Toraman FN, Kizilay F, Özdemir Ö, Cetinkaya V. Effect of strength and endurance training on cognition in older people. J Sports Sci Med. 2005;4(3):300.

33. Krist L, Dimeo F, Keil T. Can progressive resistance training twice a week improve mobility, muscle strength, and quality of life in very elderly nursing-home residents with impaired mobility? A pilot study. Clin Interv Aging. 2013;8:443.

34. Ojagbemi A, Akin-Ojagbemi N. Exercise and quality of life in dementia: a systematic review and meta-analysis of randomized controlled trials. JAG. 2017;1:0733464817693374.

35. Zwarenstein $\mathrm{M}$, Treweek S, Gagnier JJ, et al. Improving the reporting of pragmatic trials: an extension of the CONSORT statement. BMJ. 2008;337:a2390.

36. American Psychiatric Association. Diagnostic and Statistical Manual of Mental Disorders. 4th ed. Washington, DC: American Psychiatric Association; 1994.

37. Folstein MF, Folstein SE, McHugh PR. "Mini-mental state": a practical method for grading the cognitive state of patients for the clinician. J Psychiatr Res. 1975;12(3):189-198.

38. Rikli RE, Jones CJ. The reliability and validity of a 6-minute walk test as a measure of physical endurance in older adults. J Aging Phys Act. 1998;6(4):363-375.

39. Verhage F. Intelligentie en leeftijd: onderzoek bij Nederlanders van twaalf tot zevenenzeventig jaar [Intelligence and age: study with Dutch people aged 12-77]. Assen: Van Gorcum; 1964. Dutch.

40. Reisberg B, Ferris SH, de Leon MJ, Crook T. The Global Deterioration Scale for assessment of primary degenerative dementia. Am J Psychiatry. 1982;139(9):1136-1139.
41. Saxton J, Kastango KB, Hugonot-Diener L, et al. Development of a Short Form of the Severe Impairment Battery. Am J Geriatr Psychiatry. 2005;13(11):999-1005.

42. Dijkstra A, Buist G, Moorer P, Dassen T. Construct validity of the nursing care dependency scale. J Clin Nurs. 1999;8(4):380-388.

43. Graessel E, Viegas R, Stemmer R, Küchly B, Kornhuber J, Donath C. The Erlangen Test of Activities of Daily Living: first results on reliability and validity of a short performance test to measure fundamental activities of daily living in dementia patients. Int Psychogeriatr. 2009; 21(1):103-112.

44. Dijkstra A, White M, Smith J. Measuring Care Dependency with the Care Dependency Scale (CDS) - A Manual. Groningen, the Netherlands: Noordelijk Centrum voor Gezondheidsvraagstukken; 2006.

45. Ettema TP, Dröes RM, de Lange J, Mellenbergh GJ, Ribbe MW. QUALIDEM: development and evaluation of a dementia specific quality of life instrument. Scalability, reliability and internal structure. Int J Geriatr Psychiatry. 2007;22(6):549-556.

46. Ettema TP, Dröes RM, de Lange J, Mellenbergh GJ, Ribbe MW. QUALIDEM: development and evaluation of a dementia specific quality of life instrument - validation. Int J Geriatr Psychiatry. 2007;22(5):424 430.

47. Bouman A, Ettema T, Wetzels R, Van Beek A, De Lange J, Dröes R. Evaluation of Qualidem: a dementia-specific quality of life instrument for persons with dementia in residential settings; scalability and reliability of subscales in four Dutch field surveys. Int J Geriatr Psychiatry. 2011;26(7):711-722.

48. ArjoHuntleigh. ARJO Guidebook for Architects and Planners: Elderly Care Facilities. ARJO Hospital Equipment. ArjoHuntleigh: Sweden; 2005.

49. Twisk JW. Applied Longitudinal Data Analysis for Epidemiology: A Practical Guide. 2nd ed. Cambridge: Cambridge University Press; 2003.

50. Steeman E, Godderis J, Grypdonck M, De Bal N, De Casterlé BD. Living with dementia from the perspective of older people: Is it a positive story? Aging Ment Health. 2007;11(2):119-130.

51. Hoogeveen FR, Caffò AO, Bos J. Assistance in maintaining a positive self-image in people with dementia. RIFP. 2015;6(2):417-431.

52. Droës RM, Van Mierlo LD, Van der Roest HG, Meiland FJ. Focus and effectiveness of psychosocial interventions for people with dementia in institutional care settings from the perspective of coping with the disease. Nonpharmacol Ther Dement. 2010;1:139-161.

53. Rijnaard M, van Hoof J, Janssen B, et al. The factors influencing the sense of home in nursing homes: a systematic review from the perspective of residents. J Aging Res. 2016;2016:6143645.

54. Klaassens M, Meijering L. Experiences of home and institution in a secured nursing home ward in the Netherlands: a participatory intervention study. J Aging Stud. 2015;34:92-102.

55. Carpenter GI, Hastie CL, Morris JN, Fries BE, Ankri J. Measuring change in activities of daily living in nursing home residents with moderate to severe cognitive impairment. BMC Geriatr. 2006;6(1):7.

56. World Health Organization. Global Recommendations on Physical Activity for Health 2010. Available from: https://www.ncbi.nlm.nih.gov/books/ NBK305057/pdf/Bookshelf_NBK305057.pdf. Accessed March 7, 2017.

57. Telenius EW, Engedal K, Bergland A. Effect of a high-intensity exercise program on physical function and mental health in nursing home residents with dementia: an assessor blinded randomized controlled trial. PLoS ONE. 2015;10(5):e0126102.

58. Logsdon RG, McCurry SM, Teri L. Evidence-based interventions to improve quality of life for individuals with dementia. Alzheimer's Care Today. 2007;8(4):309-318.

59. Venturelli M, Scarsini R, Schena F. Six-month walking program changes cognitive and ADL performance in patients with Alzheimer. Am J Alzheimers Dis. 2011;26(5):381-388.

60. Dichter MN, Quasdorf T, Schwab CGG, et al. Dementia care mapping: effects on residents' quality of life and challenging behavior in German nursing homes. A quasi-experimental trial. Int Psychogeriatr. 2015; 27(11):1875-1892.

61. Martin M, Schneider R, Eicher S, Moor C. The Functional Quality of Life (fQOL)-model: a new basis for quality of life-enhancing interventions in old age. GeroPsych. 2012;25(1):33. 
Clinical Interventions in Aging

\section{Publish your work in this journal}

Clinical Interventions in Aging is an international, peer-reviewed journal focusing on evidence-based reports on the value or lack thereof of treatments intended to prevent or delay the onset of maladaptive correlates of aging in human beings. This journal is indexed on PubMed Central, MedLine,

CAS, Scopus and the Elsevier Bibliographic databases. The manuscript management system is completely online and includes a very quick and fair peer-review system, which is all easy to use. Visit http://www.dovepress. $\mathrm{com} /$ testimonials.php to read real quotes from published authors.

Submit your manuscript here: http://www.dovepress.com/clinical-interventions-in-aging-journal 